\title{
Long-term outcomes of Graves' disease in children and adolescents receiving antithyroid drugs
}

\author{
Jung Gi Rho, \\ Change Dae Kum, \\ Young Jun Seo, \\ Young Suk Shim, \\ Hae Sang Lee, \\ Jin Soon Hwang
}

Department of Pediatrics, Ajou University Hospital, Ajou University School of Medicine, Suwon, Korea
Received: 18 December, 2020

Revised: 30 January, 2021

Accepted: 8 March, 2021

Address for correspondence: Hae Sang Lee

Department of Pediatrics, Ajou University Hospital, Ajou University School of Medicine, 164 World cupro, Yeongtong-gu, Suwon 16499, Korea

Email: seaon98@ajou.ac.kr https://orcid.org/0000-0002-96844042
Purpose: Antithyroid drugs (ATDs) are primarily used as an initial treatment in pediatric patients with Graves' disease (GD). We aimed to investigate the long-term outcomes in pediatric GD patients receiving ATDs.

Methods: Retrospective data from a single center were collected from April 2003 to July 2020. A total of 98 children and adolescents aged 2-16 years diagnosed with GD and receiving ATDs was enrolled. We investigated the factors correlated with remission by comparing children who achieved remission after 5 years and those with persistent disease.

Results: The study included 76 girls $(77.6 \%)$ and 22 boys (22.4\%). During the 5-year follow-up period, 18 children (18.3\%) maintained remission, ATDs could not be discontinued in 74 patients (75.5\%), and relapse occurred in 6 patients (6.2\%). The remission group had significantly lower thyroid-stimulating hormone-binding inhibitory immunoglobulin (TBII) level at diagnosis $(P=0.002)$ and 3 months $(P=0.002)$, 1 year $(P=0.002), 2$ years $(P \leq 0.001), 3$ years $(P \leq 0.001), 4$ years $(P \leq 0.001)$, and 5 years $(P \leq 0.001)$ after ATD treatment than did the nonremission group. The remission group also had a shorter time for TBIl normalization after ATD treatment $(P \leq 0.001)$. Multiple logistic regression analysis showed that the time to TBII normalization (cutoff time $=2.35$ years) was related to $\mathrm{GD}$ remission (odds ratio, 0.596; 95\% confidence interval, 0.374-0.951).

Conclusion: TBII level and time to TBII normalization after ATD treatment can be used to predict remission in pediatric GD patients.

Keywords: Graves' disease, Thyroid-stimulating hormone-binding inhibitory immunoglobulin, Antithyroid drugs, Remission

\section{Highlights}

TBII level at diagnosis, and during ATD treatment in pediatric Graves' disease (GD) patients were lower in the remission group. And time to TBII normalization after ATD treatment was shorter in the remission group. Therefore, measurement of TBII level during ATD treatment can be used to predict remission in pediatric GD patients.

\section{Introduction}

Hyperthyroidism is a disease associated with clinical symptoms such as goiter and ophthalmopathy due to excessive production of thyroid hormones. The most common cause of hyperthyroidism in children is Graves' disease (GD), accounting for approximately 95\% of cases in Korea. ${ }^{1)}$ GD is caused by organ-specific autoimmunity associated with thyroid-stimulating hormone (TSH) receptor-stimulating antibodies and is linked to genetic predisposition and environmental factors. ${ }^{2,3)}$ Stimulation of the thyroid gland due to autoimmunity causes follicular cell hypertrophy and an increase in the number of follicular 
cells, resulting in increased production of thyroid hormones, diffuse goiter, and ophthalmopathy.

GD treatment aims to maintain normal thyroid function and prevent hyperthyroidism recurrence. However, the optimal GD treatment in children is controversial. Pediatric GD treatments include antithyroid drug (ATD) treatment, which suppresses thyroid hormone production, and ablative treatments (i.e., radioiodine therapy or surgery). ATDs are primarily used as an initial treatment in children because they are convenient and have the advantage of preventing permanent hypothyroidism, while ablative therapy is considered in cases of hyperthyroid relapse. However, the rate of GD remission with ATD treatment alone is low. In addition, clinical guidelines regarding the timing of ATD treatment discontinuation in children and adolescents have not been established. Hence, we investigated the remission rate following ATD treatment in pediatric patients with GD. We also determined the predictive factors for GD remission.

\section{Materials and methods}

\section{Subjects}

We retrospectively analyzed the clinical data from 98 children and adolescents diagnosed with GD (22 boys and 76 girls) who were followed for more than 5 years at Ajou University Hospital between March 2003 and July 2020. All participants were younger than 18 years at the time of diagnosis and showed evidence of autoimmune disease. ATD treatment was initiated in all patients after being diagnosed with GD based on medical history, clinical symptoms, examination findings (tachycardia, weight loss, increased sweating, goiter, and ophthalmopathy), thyroid function test results, and thyroid autoantibody test results.

\section{Patient evaluation and outcome measures}

Age, sex, height, weight, body mass index (BMI), pubertal status, pulse rate (tachycardia: pulse rate $\geq 90 \mathrm{BPM}$ ), goiter or ophthalmopathy, and family history of thyroid disease were investigated upon initial GD diagnosis. BMI $\left(\mathrm{kg} / \mathrm{m}^{2}\right)$ was calculated from the weight and square of the height, and the BMI standard deviation score (SDS) was calculated using data from the 2007 Korean National Growth Chart. ${ }^{5}$ Pubertal development was determined according to Tanner stage (prepuberty vs. puberty) based on breast development associated with a sexual maturity rating $\geq 2$ for females and testicular enlargement to at least $4 \mathrm{~mL}$ in volume for males. Goiter was classified using the World Health Organization criteria: grade 0 , no goiter; grade 1, mild goiter; and grade 2, severe goiter. ${ }^{6}$ At the time of first diagnosis, thyroid function tests were performed by measuring total triiodothyronine (T3), free thyroxine (fT4), and TSH serum levels using immunoradiometric assays (Immunotech s.r.o., Praha, Czech Republic). Tests were performed every $2-4$ weeks until thyroid function normalized and every 2-3 months thereafter. In addition, levels of thyroid autoantibodies, antithyroglobulin antibodies (ATAs), and antimicrosomal antibodies (AMAs) were measured using radioimmunoassay (Immunoradiometric assay, Cobra II gamma counter, Packard, Conroe, TX, USA). TSH-binding inhibitory immunoglobulin (TBII) level was measured using a radioreceptor assay (TSH Rezak, Medipan Diagnostica, Dahlewitz, Germany; normal range, 0.0-9.0 U/L) before June 2011. After July 2011, TBII level was measured using a TRAK human radioreceptor assay (BRAHMS, Diagnostica AG, Hennigsdorf, Germany; normal range, 0.0-1.5 U/L); a correction formula was used to standardize TRAb levels with a standard range of $0.0-1.5 \mathrm{U} / \mathrm{L}^{7)}$ After initiating ATD treatment, the interval to euthyroid status and time to TBII normalization were investigated. In addition, complete blood count and liver function tests were performed at intervals of 2-3 months during the dosing period to identify any side effects associated with ATD treatment. After GD diagnosis, the patient was administered 5-7 mg/kg propylthiouracil (PTU) 3 times a day or $0.5-0.7 \mathrm{mg} / \mathrm{kg}$ methimazole (MMZ) once a day. ATD treatment was discontinued if normal thyroid function was maintained and TBII level remained normalized for longer than 1 year. Remission was defined as normal thyroid function for at least 12 months after discontinuing ATD treatment. Patients were considered to have relapsed if they tested positive for TBII, showed increased T3 and/or fT4 levels and decreased TSH level, and showed clinical symptoms corresponding to hyperthyroidism that required resumption of ATD treatment during the total follow-up period.

\section{Patient assignment}

The participants were divided into 2 groups: patients who maintained remission were categorized into the remission group, while those who had persistent disease were categorized into the nonremission group.

\section{Statistical analysis}

We compared results of the thyroid function tests and thyroid antibody tests (ATA, AMA, and TBII) at the time of diagnosis, at 3 months, and at 1,2, 3, 4, and 5 years after ATD treatment between patients in the remission and nonremission groups. IBM SPSS Statistics ver. 25.0 (IBM Co., Armonk, NY, USA) was used to perform statistical analyses. Statistical significance of the findings between the remission and nonremission groups was assessed using the Mann-Whitney $U$-test for continuous variables and the chi-square test for categorical variables. Multiple logistic regression analysis was performed to determine the factors predicting remission, and odds ratios (ORs) and 95\% confidence intervals (CIs) were determined. In model 1, the unadjusted OR and 95\% CI were calculated using multiple logistic regression without adjusting for confounders. In model 2, the adjusted OR and 95\% CI were determined 
using multiple logistic regression after adjusting for age, sex, and BMI SDS. In model 3, multiple logistic regression analysis was performed to determine the statistical significance between remission and clinical variables of age, sex, BMI SDS, pubertal stage, goiter, exophthalmos, family history of thyroid disease, TBII level, and time to TBII normalization. We performed receiver operating characteristic (ROC) analyses to evaluate the optimal cutoff time for TBII normalization after ATD treatment in predicting remission. Optimal cutoff time was estimated by calculating Youden index (J, maximum [sensitivity+specificity -1]). A $P$-value of $<0.05$ was considered statistically significant.

\section{Results}

The mean age of the participants was $11.57 \pm 3.13$ years. Of the total 98 patients, $76(77.6 \%)$ were female and $22(22.4 \%)$ were male. The mean BMI SDS was $0.06 \pm 1.01$. We observed goiter in 95 participants (stages 1 [67.3\%] and 2 [29.6\%]) and ophthalmopathy in $47(47.9 \%)$ participants. In total, 32 participants (32.7\%) had a family history of thyroid disease. After initiating ATD treatment, the average time to euthyroid status was $0.43 \pm 0.43$ months, and the average time to TBII normalization was $3.29 \pm 2.70$ years.

During the 5-year follow-up period, 18 participants (18.3\%; 15 females and 3 males) maintained remission without relapse. Table 1 shows the clinical characteristics at diagnosis according to remission. There were no significant differences between the 2 groups with respect to age, sex, or BMI SDS at the time of GD onset. The average time to discontinue ATD treatment in the remission group was $2.94 \pm 1.01$ years. In the nonremission group, 74 patients could not discontinue ATD treatment and relapse occurred within 5 years in 6 patients $(6.2 \%)$ after discontinuing ATD treatment.

Changes in thyroid function and thyroid antibody test results in the remission and nonremission groups over 5 years are summarized in Table 2. Regarding the thyroid function test results, fT4 (3.22 mIU/L vs. $3.77 \mathrm{mIU} / \mathrm{L}, P=0.024)$, T3 (387.61 $\mathrm{mIU} / \mathrm{L}$ vs. $400.69 \mathrm{mIU} / \mathrm{L}, P=0.032)$, and TSH $(0.03 \mathrm{mIU} / \mathrm{L}$ vs. $0.14 \mathrm{mIU} / \mathrm{L}, P=0.034$ ) levels at diagnosis were significantly lower in the remission group than in the nonremission group.

TBII, AMA, and ATA positivity was observed in $100.0 \%$, $74.5 \%$, and $79.6 \%$ of participants, respectively. There were no significant differences in ATA or AMA level between the 2 groups over 5 years. However, TBII level at diagnosis (9.37 U/ $\mathrm{L}$ vs. $18.88 \mathrm{U} / \mathrm{L}, P=0.002)$, at 3 months $(5.46 \mathrm{U} / \mathrm{L}$ vs. $13.09 \mathrm{U} /$ $\mathrm{L}, P=0.002)$, and at 1 year $(2.42 \mathrm{U} / \mathrm{L}$ vs. $7.67 \mathrm{U} / \mathrm{L}, P=0.002), 2$ years (1.76 U/L vs. $2.30 \mathrm{U} / \mathrm{L}, P \leq 0.001), 3$ years $(1.34 \mathrm{U} / \mathrm{L}$ vs. 8.39 $\mathrm{U} / \mathrm{L}, P \leq 0.001), 4$ years $(1.78 \mathrm{U} / \mathrm{L}$ vs. $7.44 \mathrm{U} / \mathrm{L}, P \leq 0.001)$, and 5 years $(1.38 \mathrm{U} / \mathrm{L}$ vs. $7.01 \mathrm{U} / \mathrm{L}, P \leq 0.001)$ after ATD treatment were significantly lower in the remission group than in the nonremission group. There was no significant difference between the 2 groups in euthyroid status after ATD treatment. However, time to TBII normalization after ATD treatment (1.65 years vs. 3.65 years, $P<0.001)$ was significantly shorter in the remission group than in the nonremission group.

Multiple logistic regression analysis was performed to evaluate the association between remission and factors related to GD, with remission as the dependent variable (Table 3). As one year to TBII normalization (years) increased, OR for remission decreased 0.639 (95\% CI, 0.456-0.894) in unadjusted

Table 1. Clinical characteristics of the study population at the time of diagnosis of Graves' disease according to the occurrence of remission during the 5-year follow-up period

\begin{tabular}{|c|c|c|c|c|}
\hline Characteristic & All participants $(n=98)$ & Remission $(n=18)$ & Nonremission $(n=80)$ & $P$-value \\
\hline Female sex & $76(77.6)$ & $15(83.3)$ & $61(76.3)$ & 0.515 \\
\hline Age (yr) & $11.57 \pm 3.13$ & $10.41 \pm 3.57$ & $11.83 \pm 2.98$ & 0.102 \\
\hline BMI SDS & $0.06 \pm 1.01$ & $-0.66 \pm 1.09$ & $-0.36 \pm 0.98$ & 0.160 \\
\hline fT4 (ng/dL) & $3.67 \pm 1.28$ & $3.22 \pm 1.49$ & $3.77 \pm 1.21$ & 0.024 \\
\hline $\mathrm{T} 3$ (ng/dL) & $398.29 \pm 215.59$ & $387.61 \pm 378.07$ & $400.69 \pm 162.11$ & 0.032 \\
\hline TSH $(\mu \mathrm{lU} / \mathrm{mL})$ & $0.12 \pm 0.71$ & $0.03 \pm 0.04$ & $0.14 \pm 0.78$ & 0.034 \\
\hline ATA $(\mathrm{U} / \mathrm{mL})$ & $524.91 \pm 958.39$ & $460.72 \pm 1113.01$ & $539.54 \pm 926.99$ & 0.911 \\
\hline $\mathrm{AMA}(\mathrm{U} / \mathrm{mL})$ & $1,828.79 \pm 1,837.23$ & $1,772.33 \pm 1983.53$ & $1,841.50 \pm 1,815.76$ & 0.960 \\
\hline TBII (U/L) & $54.71 \pm 77.08$ & $31.27 \pm 34.08$ & $60.00 \pm 83.01$ & 0.089 \\
\hline Puberty & & & & 0.865 \\
\hline Prepubertal & $23(23.5)$ & $5(27.8)$ & $18(22.5)$ & \\
\hline Tanner Ilb, or Testis $\geq 4 \mathrm{~mL}$ & $75(76.5)$ & $13(72.2)$ & $62(77.5)$ & \\
\hline Tachycardia & $80(81.6)$ & $11(61.1)$ & $69(86.3)$ & 0.031 \\
\hline Goiter (grade 2) & $29(29.6)$ & $4(22.2)$ & $25(31.3)$ & 0.637 \\
\hline Exophthalmos & $47(47.9)$ & $8(44.4)$ & $39(48.8)$ & 0.945 \\
\hline Family history of thyroid disease & $32(32.7)$ & $6(33.3)$ & $26(32.5)$ & $>0.999$ \\
\hline Time to euthyroid (mo) & $0.43 \pm 0.43$ & $0.35 \pm 0.26$ & $0.45 \pm 0.46$ & 0.609 \\
\hline Time to normal TBII (yr) & $3.29 \pm 2.70$ & $1.65 \pm 1.38$ & $3.65 \pm 2.77$ & $<0.001$ \\
\hline
\end{tabular}

Values are presented as number (\%) of mean \pm standard deviation.

BMI, body mass index; SDS, standard deviation score; fT4, free thyroxine; T3, triiodothyronine; TSH, thyroid-stimulating hormone; ATA, antithyroglobulin antibody; AMA, antimicrosomal antibody; TBII, thyroid-stimulating hormone-binding inhibitory immunoglobulin. 
Table 2. Changes in the levels of serum free thyroxine, triiodothyronine, thyroid-stimulating hormone, and thyroid-stimulating hormone-binding inhibitory immunoglobulin during the 5-year follow-up period according to the remission or nonremission of Graves' disease

\begin{tabular}{|c|c|c|c|}
\hline Variable & Remission & Nonremission & $P$-value \\
\hline \multicolumn{4}{|l|}{ At diagnosis } \\
\hline fT4 (ng/dL) & $3.22 \pm 1.49$ & $3.77 \pm 1.21$ & 0.024 \\
\hline T3 (ng/dL) & $387.61 \pm 378.07$ & $400.69 \pm 162.11$ & 0.032 \\
\hline TSH ( $\mu \mathrm{IU} / \mathrm{mL})$ & $0.03 \pm 0.04$ & $0.14 \pm 0.78$ & 0.034 \\
\hline TBII (U/L) & $9.37 \pm 8.87$ & $18.88 \pm 19.30$ & 0.002 \\
\hline \multicolumn{4}{|l|}{ At 3 months } \\
\hline fT4 (ng/dL) & $1.35 \pm 0.66$ & $1.42 \pm 0.69$ & 0.653 \\
\hline T3 (ng/dL) & $135.56 \pm 40.00$ & $159.43 \pm 60.77$ & 0.143 \\
\hline TSH ( $\mu \mathrm{IU} / \mathrm{mL})$ & $5.66 \pm 12.39$ & $2.59 \pm 9.41$ & 0.872 \\
\hline TBII (U/L) & $5.46 \pm 7.41$ & $13.09 \pm 8.87$ & 0.002 \\
\hline \multicolumn{4}{|l|}{ At 1 year } \\
\hline fT4 (ng/dL) & $1.21 \pm 0.24$ & $1.39 \pm 0.50$ & 0.102 \\
\hline T3 (ng/dL) & $143.61 \pm 36.94$ & $145.35 \pm 46.98$ & 1.000 \\
\hline TSH ( $\mu \mathrm{IU} / \mathrm{mL})$ & $1.98 \pm 0.46$ & $2.96 \pm 5.27$ & 0.483 \\
\hline TB\| (U/L) & $2.42 \pm 4.41$ & $7.67 \pm 11.59$ & 0.002 \\
\hline \multicolumn{4}{|l|}{ At 2 years } \\
\hline fT4 (ng/dL) & $1.22 \pm 0.35$ & $1.37 \pm 0.44$ & 0.265 \\
\hline T3 (ng/dL) & $126.17 \pm 34.68$ & $133.69 \pm 22.28$ & 0.916 \\
\hline TSH ( $\mu \mathrm{IU} / \mathrm{mL})$ & $4.79 \pm 8.18$ & $2.05 \pm 2.51$ & 0.025 \\
\hline TBII (U/L) & $1.76 \pm 7.05$ & $2.30 \pm 11.26$ & $<0.001$ \\
\hline \multicolumn{4}{|l|}{ At 3 years } \\
\hline fT4 (ng/dL) & $1.24 \pm 0.25$ & $6.86 \pm 46.55$ & 0.095 \\
\hline T3 (ng/dL) & $122.53 \pm 17.89$ & $148.73 \pm 76.91$ & 0.599 \\
\hline $\mathrm{TSH}(\mu \mathrm{l} \mathrm{U} / \mathrm{mL})$ & $2.70 \pm 3.03$ & $2.23 \pm 6.04$ & 0.160 \\
\hline TBII (U/L) & $1.34 \pm 1.98$ & $8.39 \pm 11.48$ & $<0.001$ \\
\hline \multicolumn{4}{|l|}{ At 4 years } \\
\hline fT4 (ng/dL) & $1.37 \pm 0.24$ & $1.53 \pm 0.68$ & 0.773 \\
\hline T3 (ng/dL) & $125.83 \pm 31.28$ & $143.96 \pm 70.05$ & 0.460 \\
\hline TSH $(\mu \mathrm{lU} / \mathrm{mL})$ & $0.96 \pm 0.72$ & $1.79 \pm 2.65$ & 0.755 \\
\hline TBII (U/L) & $1.78 \pm 2.61$ & $7.44 \pm 10.83$ & $<0.001$ \\
\hline \multicolumn{4}{|l|}{ At 5 years } \\
\hline fT4 (ng/dL) & $1.31 \pm 0.20$ & $1.56 \pm 0.63$ & 0.118 \\
\hline T3 (ng/dL) & $120.33 \pm 33.14$ & $141.46 \pm 72.40$ & 0.317 \\
\hline TSH ( $\mu \mathrm{lU} / \mathrm{mL})$ & $1.44 \pm 1.41$ & $1.52 \pm 1.84$ & 0.302 \\
\hline TBII (U/L) & $1.38 \pm 2.11$ & $7.01 \pm 10.72$ & $<0.001$ \\
\hline Time to euthyroid (mo) & $0.35 \pm 0.26$ & $0.45 \pm 0.46$ & 0.605 \\
\hline Time to normal TBII (yr) & $1.65 \pm 1.38$ & $3.65 \pm 2.77$ & $<0.001$ \\
\hline
\end{tabular}

Values are presented as mean \pm standard deviation.

fT4, free thyroxine; T3, triiodothyronine; TSH, thyroid-stimulating hormone; TBII, thyroid-stimulating hormone-binding inhibitory immunoglobulin.

model 1 . This relationship remained statistically significant after adjusting for confounding factors in models 2 and 3 . The time to TBII normalization (years) showed ORs for remission of 0.608 (95\% CI, 0.425-0.870) and 0.596 (95\% CI, 0.374-0.951) in models 2 and 3, respectively.

According to the ROC curve analysis to evaluate optimal cutoff time for TBII normalization after ATD treatment in predicting remission, TBII normalization of 2.35 years
Table 3. Multiple logistic regression analysis of association between remission and clinical variables

\begin{tabular}{lccc}
\hline \multirow{2}{*}{ TBII } & \multicolumn{3}{c}{ OR $(95 \% \mathrm{Cl})$} \\
\cline { 2 - 4 } & Mode 1 & Mode 2 & Mode 3 \\
\hline At diagnosis (U/L) & 0.953 & 0.946 & 0.990 \\
& $(0.906-1.001)$ & $(0.896-0.998)$ & $(0.902-1.086)$ \\
3 Months (U/L) & 0.922 & 0.985 & 0.959 \\
& $(0.853-0.995)$ & $(0.971-0.998)$ & $(0.798-1.152)$ \\
1 Year (U/L) & 0.828 & 0.819 & 1.024 \\
& $(0.680-1.010)$ & $(0.671-1.001)$ & $(0.860-1.219)$ \\
2 Years (U/L) & 0.848 & 0.838 & 0.927 \\
& $(0.687-1.048)$ & $(0.674-1.040)$ & $(0.686-1.252)$ \\
3 Years (U/L) & 0.711 & 0.677 & 0.815 \\
& $(0.509-0.993)$ & $(0.472-0.972)$ & $(0.576-1.154)$ \\
4 Years (U/L) & 0.812 & 0.806 & 0.902 \\
& $(0.653-1.008)$ & $(0.647-1.003)$ & $(0.634-1.282)$ \\
5 Years (U/L) & 0.757 & 0.749 & 0.949 \\
& $(0.569-1.007)$ & $(0.563-0.997)$ & $(0.653-1.379)$ \\
Time to normal TBII (yr) & 0.639 & 0.608 & 0.596 \\
& $(0.456-0.894)$ & $(0.425-0.870)$ & $(0.374-0.951)$ \\
\hline OR, odds ratio; Cl, con
\end{tabular}

OR, odds ratio; $\mathrm{Cl}$, confidence interval; TBll, thyroid- stimulating hormone-binding inhibitory immunoglobulin.

Model 1: Statistical significance between remission and clinical variables was determined using multiple logistic regression with no adjustments. Model 2: Statistical significance between remission and clinical variables was determined using multiple logistic regression after adjusting for age, sex, and body mass index (BMI) standard deviation score (SDS). Model 3: Statistical significance between remission and clinical variables, including age, sex, BMI SDS, pubertal stage, goiter, exophthalmos, family history of thyroid disease, TBII levels, and time to TBII normalization was determined using multiple logistic regression analysis.

correlated with remission with an area under the curve of 0.766 , $66.3 \%$ sensitivity, and $88.9 \%$ specificity.

\section{Discussion}

In our study on pediatric GD patients, the remission rate was 18.2\% with ATD treatment alone during the 5-year follow-up period. In comparison between the remission and nonremission groups, time to TBII normalization was significantly shorter in the remission group than in the nonremission group. In adult GD patients, the remission rate after $1-2$ years of ATD treatment is $40 \%-50 \%$ and the recurrence rate is $30 \%-70 \%{ }^{8}$ Magri et al. ${ }^{9)}$ reported that, during a 5 -year follow-up period, approximately $54.1 \%$ of adults receiving ATD treatment showed remission and GD recurred in $11.2 \%$ of adults. Although the period for ATD treatment has not been established, studies have reported that the 4 - and 5-year remission rates following 2 years of pediatric ATD treatment are $25 \%$ and $50 \%$, respectively. ${ }^{10,11)}$ The incidence of relapse is variable, and recurrence occurs in $3 \%-47 \%$ of pediatric GD patients after discontinuing ATD treatment. ${ }^{1,12)}$ Lee et al. ${ }^{13)}$ reported that $6.3 \%$ of pediatric GD patients achieved remission after 3 years, $16.4 \%$ achieved remission after 4 years, 29.4\% achieved remission after 5 years, 
and $55.8 \%$ achieved remission after 6 years post-ATD treatment. In the present study, 18 children (18.3\%) achieved remission during the 5-year observation period, and medications could not be discontinued in the remaining 74 patients (75.5\%) during this period. The relatively small number of subjects and the selection bias inherent to the retrospective study design might explain why the observed remission rate was lower than that observed in other studies. Relapse occurred in 6 of 24 patients (25\%) who discontinued ATD treatment during the 5-year follow-up period. Since the remission rate with ATD treatment alone is low, establishing a treatment plan based on reliable predictive factors for remission is warranted.

Several studies have focused on the identification of predictive factors for remission. Glaser et al. ${ }^{14,15)}$ concluded that a higher BMI SDS, smaller goiter size, lower initial T3 level, and older age were associated with remission. In addition, several other studies proposed that higher AMA level, higher TSH level, and lower ATA level are correlated with remission. ${ }^{16,17)}$ However, since most of these studies were retrospective and not large-scale or long-term studies, reliable predictive factors for remission in pediatric patients with GD remain controversial. There were no significant differences in sex, age, BMI SDS, goiter size, fT4, T3, ATA, or AMA level between the remission and nonremission groups in this study. However, the remission group had significantly lower TBII level at diagnosis and 3 months and at 1,2,3,4, and 5 years after ATD treatment relative to the nonremission group. We also found that the remission group had a significant positive relationship with shorter time to TBII normalization. Through ROC analysis, we identified that TBII normalization of 2.35 years was predictive of remission in pediatric GD patients after ATD treatment.

TBII measurement is an essential test for GD diagnosis, and TBII levels are estimated based on thyroid-stimulating or blocking antibody levels. Several studies in adults have indicated that TBII levels can predict GD remission. ${ }^{18-20)}$ In addition, Lee et al. ${ }^{13)}$ and Gastaldi et al. ${ }^{7)}$ demonstrated that rapid TBII normalization is significantly correlated with remission in pediatric GD patients. However, Zimmermann-Belsing et al. ${ }^{21)}$ concluded that TBII level is not an appropriate predictor of remission in GD patients.

Our study has some limitations. First, bias might have been introduced as the same therapeutic agent was not initially used. We used PTU only when thyrotoxicosis symptoms were severe and switched to MMZ when patient thyroid function normalized. After a possible occurrence of PTU-induced hepatitis was observed, MMZ was administered. Second, since the kit used for measuring TBII changed, we used a correction formula to consider different reference ranges. Finally, due to the retrospective cross-sectional design of the study, temporal and causal associations could not be established. Further studies with larger sample sizes and prospective and longitudinal designs are needed to confirm our findings.

In conclusion, the time to TBII normalization after ATD treatment can be used as an important predictive factor for maintenance of remission in pediatric patients with GD.
Reliable predictive factors for remission can help determine whether ATD treatment alone is sufficient or if early ablative treatment is needed. Therefore, measurement of TBII level during treatment can help establish a proper treatment plan for each patient.

\section{Ethical statement}

The study design was approved by the Institutional Review Board of Ajou University Hospital (AJIRB-MED-MDB-20-377), and the requirement for written informed consent was waived due to the retrospective nature of the study.

\section{Conflict of interest}

No potential conflict of interest relevant to this article was reported.

\section{Funding}

This research received no specific grant from any funding agency in the public, commercial, or not-for-profit sectors.

\section{References}

1. Song SM, Youn JS, Ko JM, Cheon CK, Choi JH, Yoo HW. The natural history and prognostic factors of Graves' disease in Korean children and adolescents. Korean J Pediatr 2010;53:585-91.

2. Weetman AP. Graves' disease. N Engl J Med 2000;343:123648.

3. Tomer Y. Mechanisms of autoimmune thyroid diseases: from genetics to epigenetics. Annu Rev Pathol 2014;9:14756.

4. Zimmerman D, Gan-Gaisano M. Hyperthyroidism in children and adolescents. Pediatr Clin North Am 1990;37: 1273-95.

5. Moon JS, Lee SY, Nam CM, Choi JM, Choe BK, Seo JW, et al. 2007 Korean National Growth Charts: review of developmental process and an outlook. Korean J Pediatr 2008;51:1-25.

6. World Health Organization. Indicators for assessing iodine deficiency disorders and their control through salt iodization. Geneva (Switzerland): World Health Organization, 1994.

7. Gastaldi R, Poggi E, Mussa A, Weber G, Vigone MC, Salerno $\mathrm{M}$, et al. Graves disease in children: thyroid-stimulating hormone receptor antibodies as remission markers. J Pediatr 2014;164:1189-94.e1.

8. Smith TJ, Hegedüs L. Graves' Disease. N Engl J Med 2016;375:1552-65.

9. Magri F, Zerbini F, Gaiti M, Capelli V, Ragni A, Rotondi $\mathrm{M}$, et al. Gender influences the clinical presentation and 
long-term outcome of Graves disease. Endocr Pract 2016;22:1336-42.

10. Lippe BM, Landaw EM, Kaplan SA. Hyperthyroidism in children treated with long term medical therapy: twentyfive percent remission every two years. J Clin Endocrinol Metab 1987;64:1241-5.

11. Gruñeiro-Papendieck L, Chiesa A, Finkielstain G, Heinrich JJ. Pediatric Graves' disease: outcome and treatment. J Pediatr Endocrinol Metab 2003;16:1249-55.

12. Kaguelidou F, Carel JC, Léger J. Graves' disease in childhood: advances in management with antithyroid drug therapy. Horm Res 2009;71:310-7.

13. Lee SH, Lee SY, Chung HR, Kim JH, Kim JH, Lee YA, et al. Remission rate and remission predictors of Graves disease in children and adolescents. Korean J Pediatr 2009;52:10218.

14. Glaser NS, Styne DM. Predictors of early remission of hyperthyroidism in children. J Clin Endocrinol Metab 1997;82:1719-26.

15. Glaser NS, Styne DM; Organization of Pediatric Endocrinologists of Northern California Collaborative Graves' Disease Study Group. Predicting the likelihood of remission in children with Graves' disease: a prospective, multicenter study. Pediatrics 2008;121:e481-8.

16. Hwang SM, Kim MS, Lee DY. Predictive factors for early response to methimazole in children and adolescents with Graves disease: a single-institute study between 1993 and 2013. Ann Pediatr Endocrinol Metab 2016;21:70-4.

17. Kim SM, Hwang JS. Remission predictors of Graves' disease in children. J Korean Soc Pediatr Endocrinol 2010;15:1005.

18. Schott M, Morgenthaler NG, Fritzen R, Feldkamp J, Willenberg HS, Scherbaum WA, et al. Levels of autoantibodies against human TSH receptor predict relapse of hyperthyroidism in Graves' disease. Horm Metab Res 2004;36:92-6.

19. Quadbeck B, Hoermann R, Roggenbuck U, Hahn S, Mann $\mathrm{K}$, Janssen $\mathrm{OE}$, et al. Sensitive thyrotropin and thyrotropinreceptor antibody determinations one month after discontinuation of antithyroid drug treatment as predictors of relapse in Graves' disease. Thyroid 2005; 15:1047-54.

20. Maugendre D, Massart C. Clinical value of a new TSH binding inihibitory activity assay using human TSH receptors in the follow-up of antithyroid drug treated Graves' disease. Comparison with thyroid stimulating antibody bioassay. Clin Endocrinol (Oxf) 2001;54:89-96.

21. Zimmermann-Belsing T, Nygaard B, Rasmussen AK, Feldt-Rasmussen U. Use of the 2nd generation TRAK human assay did not improve prediction of relapse after antithyroid medical therapy of Graves' disease. Eur J Endocrinol 2002;146:173-7. 